\title{
La política de seguridad ciudadana en Holanda: Traficando con el miedo.
}

\author{
René van Swaaningen iD \\ Universidad Erasmus de Rotterdam (Holanda) ${ }^{1}$
}

\author{
RESUMEN
}

En la literatura criminólogica internacional Holanda es generalmente caracterizado como un país tolerante y liberal: permisivo hacia muchos vicios, amistoso con los extranjeros y bendecido con un clima penal suave. Todo esto se percibe ahora como la indulgencia malentendida del pasado, indulgencia que nos ha llevado a una situación con problemas que se nos van de las manos. Con este supuesto pasado presentado como un error, Holanda en los últimos años se ha convertido en un país confundido, intolerante, punitivo y que está obsesionado con la seguridad. En este respecto, analizaremos como las ideas inclusivas sobre prevención local del delito se convirtieron en políticas excluyentes de inseguridad ciudadana. Aunque en las consecuencias existen paralelos con otros países europeos, particularmente con el Reino Unido, los factores que explican el porqué de este giro en Holanda son un tanto diferentes. El aumento del racismo contra minorías islámicas, que se desarrolló tras el ataque a las Torres Gemelas en Nueva York y el asesinato de un líder populista de derechas y un director de cine en Ámsterdam, ha sido un catalizador importante del nacimiento de una cultura del control guiada por el pánico. Otros factores que hay que considerar son el papel de los políticos populistas y los periodistas que en la actualidad actúan como "traficantes del miedo" y en los problemas de identidad que la "gente ordinaria" confronta como resultado de la globalización de la economía y la erosión del sector publico.

Palabras claves: seguridad ciudadana, miedo al delito, política criminal, populismo punitivo

\section{ABSTRACT}

In the international criminological literature the Netherlands is generally characterised as a tolerant, liberal country: permissive towards many vices, foreigner-friendly and blessed with a mild penal climate. All this is now seen as a misplaced leniency of the past, because of which we are now confronted with problems we cannot handle anymore. With this alleged past portrayed as a mistake, the Netherlands has over the last few years turned into a rather confused, intolerant, punitive country that is obsessed with safety. In this respect, we will analyse the development of inclusive ideas about a local prevention of crime into a merely exclusive politics of public safety. Though in its actual consequences the parallels with other European countries, particularly with Britain, are quite striking, the underlying factors of why this shift took place in the Netherlands are somewhat different. The increased islamophobia, following the attack on the Twin Towers in New York and the murder of Rightwing populist leader and a film director in Amsterdam has been an important catalyst of the emergence of a panic struck culture of control. Other factors to be analysed are the role of populist politicians and journalists who currently act as 'traffickers in fear' and the identity-problems 'ordinary people' face after the globalisation of the economy and the erosion of the public domain.

Keywords: community safety, fear of crime, crime policy, punitive populism

\section{Introducción}

En la literatura criminológica internacional generalmente se caracteriza a Holanda como un país liberal y tolerante: permisivo para con muchos vicios, amable con el extranjero y bendecido con un suave clima penal. Hoy en día, esta imagen parece estar tan desgastada como lo están las postales de capas pintorescas, molinos de viento y tulipanes. La autocomplacencia con nuestro "modelo de país", en el que todos los problemas sociales se abordan de manera bien planeada y pragmática, tropieza actualmente con el desdén. Una llamada 'indulgencia malentendida' del pasado nos

\footnotetext{
${ }^{1}$ Quiero agradecer a Maribel Narváez la traducción del inglés de este artículo y, a Juanjo Medina y a un revisor anónimo de esta revista los comentarios que efectuaron sobre una versión anterior.
} 
habría enfrentado a problemas que ya no podemos manejar. Durante la última década y muy especialmente durante los últimos años - Holanda se ha convertido en un país bastante intolerante, xenófobo y punitivo.

Cuando David Downes (1988) escribió su famoso libro sobre la política criminal holandesa Contrasts in Tolerance, justo vivíamos en los últimos años de la era caracterizada por el "pragmatismo de principios" que se consideraba "tolerancia" (van Swaaningen, 1998). En ese libro Downes señalaba las consecuencias negativas que tendría la política antidroga holandesa orientada principalmente hacia la asistencia y la salud pública, ya que posiblemente marcaría los límites de la tolerancia. Ciertamente es verdad que ahora la justicia penal juega un papel bastante más central en el control de drogas del que antes jugaba - principalmente en el ámbito del debate político sobre el crimen organizado. Además, un símbolo importante, en este sentido, de la tolerancia holandesa, los llamados "coffeeshops", en los que el cannabis y productos derivados pueden comprarse y usarse (casi) legalmente, están perdiendo apoyo político y su número ha decrecido en modo bastante drástico. Con todo, el verdadero límite de la tolerancia ha llegado de una dirección muy distinta. En el ámbito de las drogas todavía existe suficiente preocupación sobre las consecuencias de las alternativas más probables - una "guerra de las drogas" al estilo americano - como para mantener una posición comparativamente más liberal. Probablemente fueron pocos quienes previeron que la tolerancia encontraría su límite en unas molestias notablemente más genéricas con las que el ciudadano que observa la ley se tropieza en las calles.

Según una encuesta de 2003, el 56\% de los ciudadanos de Rotterdam considera que los grupos de jóvenes, los adictos a las drogas, los mendigos y vagabundos constituyen la principal molestia en el ámbito público. Se dice incluso, que su conducta en algunas ocasiones agresiva, intimidatoria e incordiante, constituye la mayor fuente de ansiedad de la población. Tomando como referencia en especial dicha encuesta las autoridades locales de Rotterdam decidieron en 2003 establecer una prohibición sobre la mendicidad $^{2}$. A primera vista puede parecer extraño que se utilice la preciosa capacidad de la policía para problemas relativamente menores como la mendicidad - precisamente unos años después de haber sido despenalizada. Sin embargo, constituye una buena ilustración de la dirección en la que la política de seguridad ciudadana holandesa se ha ido moviendo durante la pasada década.

\section{Una perspectiva comparativa}

Si nos fijamos en la política de seguridad ciudadana de varios países europeos podemos encontrar evoluciones bastante similares (Hebberecht \& Duprez, 2002). Autores, de diferente procedencia, hablan de un giro en la orientación de sus políticas nacionales de seguridad ciudadana ocurrido en torno a la mitad de los noventa. La lucha contra el comportamiento antisocial en las calles y contra otras acciones incívicas se presenta como nuevo centro de interés, clave de las políticas de seguridad ciudadana, en no pocos lugares. La idea de que la policía puede determinar bajo qué condiciones diferentes instituciones deben cooperar, también está muy extendida. Por su parte, la fuerza motriz que se encuentra tras estos desarrollos parece ser bastante similar en diferentes países europeos: el miedo al delito. Además, en muchos casos tales sensaciones parecen tener poco que ver con los índices reales de criminalidad. En prácticamente todos los países europeos el ámbito del sistema de justicia penal se está expandiendo bajo los mismos parámetros. Ya no se orienta hacia la lucha contra el

\footnotetext{
${ }^{2}$ Fuente: Diario NRC Handelsblad, edición del 11 de junio de 2003.
} 
delito, sino que lo hace más bien hacia la lucha contra las molestias bastante más genéricas en los espacios públicos. En toda Europa ya casi no puede vislumbrarse la tradicional distinción entre control del delito y orden público.

Específicamente en la literatura anglosajona existen muy buenos análisis del modo en que el delito y la seguridad se han convertido en los temas estrella durante las elecciones siendo el libro de David Garland The Culture of Control (2001), probablemente, el ejemplo más influyente. Con todo, y esta es la principal crítica que puede esgrimirse contra el trabajo de Garland, si contemplamos las circunstancias específicas que han influido sobre la emergencia de una "cultura del control" podemos percatarnos de grandes diferencias. Durante un seminario que organicé en Rotterdam, pedí a varios académicos europeos que describiesen el evento que más había influido en el discurso sobre seguridad y su contenido en el nuevo milenio. Per Stangeland (2004) describió los efectos del ataque con bombas en Madrid el 11 de marzo de 2004 en la política española de seguridad. Sophie Body-Gendrot (2004) señaló el pánico moral principalmente sobre norteafricanos- a bandas de jóvenes como la cuestión que ha dominado el debate sobre seguridad en Francia; y Magnus Hörnqvist (2004) se refirió a cómo el debate sobre la lucha contra el terrorismo ha desdibujado la diferencia entre control del delito y guerra en Suecia, de modo que el debate sobre seguridad se ha transformado en un debate sobre control. Este es un artículo sobre el caso holandés. En él se registran muchas similitudes con lo ocurrido en otros lugares, pero también, otras tantas diferencias.

Antes de dar propiamente comienzo a nuestro análisis, tenemos que decir algo sobre el lugar en que se localizan las políticas de seguridad. Las políticas penales de carácter general reciben orientación desde el ámbito nacional pero, de hecho, el miedo contra las molestias e inseguridades se vivencia en el ámbito local. Por ello, la literatura internacional sobre seguridad habla con frecuencia de la gestión local del delito y la inseguridad (Crawford, 2002; Hughes \& Adams, 2002; Newburn \& Sparks, 2004). Los problemas de ámbito local también son los principales responsables de la hiperpolitización del delito y de la inseguridad en el ámbito nacional. Mientras que gran parte de la literatura existente se centra, o bien en el ámbito local, o bien en el nacional, este artículo trata principalmente de la relación entre ellos. Intenta analizar algunos de los más profundos trasfondos políticos y culturales de la gobernabilidad a través del -antes que del-delito y de la inseguridad en ambos ámbitos.

\section{El surgimiento del discurso de la seguridad}

Durante mucho tiempo todo parecía muy claro: se producía un delito, la policía intentaba atrapar al culpable, el fiscal iniciaba un proceso penal y el juez determinaba si el acusado era o no culpable y qué castigo merecía. Todos estos hechos incontestables de la justicia penal empezaron a desmoronarse a mitad de los setenta. Ya no estaba claro qué era exactamente un delito debido a que este concepto incierto había sido desafiado primero por los académicos del etiquetamiento y luego, de manera aún más radical, por los abolicionistas. La policía empezó a invertir más en mantener mejores relaciones con la ciudadanía y en resolver conflictos, que en dedicarse en exclusiva a cazar delincuentes. Con el fuerte incremento del número de casos, los fiscales decidían sobre tantas cuestiones -llegando incluso autónomamente a desviar casos del proceso penalque gradualmente se fueron convirtiendo en una especia de quasi jueces. ¿Y qué pasó con los jueces? Bueno, los jueces básicamente siguieron haciendo lo que siempre habían hecho. No obstante, las decisiones más importantes ya no se tomaban en el tribunal, sino en fases anteriores del proceso penal. El juez debía entonces tener en cuenta los 
intereses de las víctimas y poseía un abanico más amplio de posibilidades sancionadoras que antes ${ }^{3}$.

A principios de los años ochenta, el debate sobre la prevención del delito tuvo su momento. Dicha política pareció dar cabida a muchas lecciones aprendidas de los abolicionistas: la justicia penal siempre llega tarde y "delito" es una etiqueta demasiado simple para capturar la complejidad de problemas a la que de hecho nos enfrentamos.

En lugar de sólo prestar atención a la represión, se debía invertir bastante más en fortalecer los vínculos, en especial de los jóvenes, con la sociedad, en aumentar el control social informal, y en hacer algo por lo que respecta a las causas (sociales) del delito. Con ello había empezado un proceso masivo que fue caracterizado por David Garland (2001) como "la estrategia de la responsabilización". Adam Crawford ha analizado de manera detallada la subsiguiente "gobernabilidad local del delito" apelando a la ciudadanía y a la presencia de socios públicos y privados. En lugar de mantener un enfoque exclusivamente penal, se propuso un enfoque de instituciones múltiples. Diferentes instituciones fueron invitadas a responsabilizarse en la lucha contra el delito a partir de sus específicas tareas y competencias. Debían también cooperar de manera más intensa entre sí. En el caso holandés, dicha cooperación involucró principalmente a las instituciones públicas, tales como la policía, la asistencia social, la asistencia juvenil, las escuelas, la vivienda municipal, y muy especialmente a las autoridades locales, antes que al sector privado (van de Bunt \& van Swaaningen, 2005). Diría que las iniciativas cívicas, jugaron comparativamente un papel marginal. Holanda es mucho más experta y puntera en cuestiones de democracia.

En los primeros años noventa, ya se veía que la expresión "prevención del delito" tenía un significado demasiado limitado para lo que de hecho estábamos haciendo. El delito era solo una causa de la ansiedad de la población, y la lucha contra sus miedos al delito y las sensaciones de inseguridad se consideraron tan importantes como la lucha contra el delito como tal, o como la presencia de otros determinantes objetivos de la inseguridad. Se intuía que un tal cambio político requeriría una completa renovación de la política urbana y social. Desde entonces en adelante, hablaríamos de una "política de seguridad integral" (uit Beijerse \& van Swaaningen, 1993). Ese cambio no fue sólo semántico. Escuchando a los políticos o a los medios de comunicación, "delito" e "inseguridad" parecen conceptos intercambiables, pero obviamente el último es mucho más amplio, subjetivo, flexible e indefinido. $\mathrm{Y}$ precisamente porque los problemas de seguridad son mucho más amplios, el control policial del delito orientado a la seguridad no sólo resulta un camino resbaladizo, sino que resulta ser un camino en el que podemos perdernos con facilidad.

Por esta razón, Willem de Haan (1995:35) advirtió inmediatamente que al convertir la "seguridad" en el concepto clave del discurso de la prevención del delito, estaríamos probablemente enturbiando más que renovando la racionalidad de la justicia penal. Nada queda explícitamente excluido del concepto de "seguridad". No obstante, la documentación policial de los primeros años noventa no habla sobre la seguridad de las mujeres, la seguridad del tráfico, el terrorismo o los desastres. Presta una especial atención a las molestias cotidianas, como incomodidades, ruidos, suciedad y degradación urbana que directamente no constituyen un problema de seguridad. Al respecto, resulta bastante ilustrativo leer el Libro Blanco del Ministerio de Justicia Hacia una sociedad más segura (Naar een veiliger samenleving). En él se sostiene que los años ochenta se caracterizaron por una malentendida tolerancia de la conducta criminogénica (jsic!) y de las molestias en el ámbito público que se habían mantenido

\footnotetext{
${ }^{3}$ Resulta imposible aquí documentar todos estos cambios. La mayoría de ellos, sin embargo, se encuentran recogidos en: van Swaaningen \& uit Beijerse, 1993.
}

Revista Española de Investigación Criminólogica

REIC AC-02-05

http://www.criminologia.net

ISSN 1696-9219 
por demasiado tiempo (MvJ, 2002:6). Se arguye que ahora las autoridades jurídico penales deben, por tanto, centrar la atención en las conductas delictivas en el ámbito público, tales como la violencia, robos en las calles, vandalismo, mala conducta en el transporte público y delincuencia juvenil (MvJ 2002:7). El término "seguridad" se interpreta cada vez en mayor medida como tolerancia cero hacia todo tipo de conductas incívicas descritas vagamente. De este modo la seguridad es tratada básicamente como un problema de orden público. Puesto que el concepto es tan poco claro, tampoco queda claro quien tiene que intervenir, o de qué modo, ni con cuanta fuerza ni en qué circunstancias.

Así: en los años setenta y ochenta, la importancia del tradicional enfoque represivo frente al delito se puso en perspectiva. Las palabras clave del nuevo enfoque fueron "prevención", "enfoque integral" y en último lugar "seguridad". Por un lado, este desarrollo facilitó un enfoque más social y estructural ante el delito. Pero, por otro lado, también hizo que la política jurídico criminal fuese muy vulnerable a dejarse llevar por los miedos y sensaciones subjetivas de los ciudadanos antes que por "hechos" en cierto modo más duros empíricamente.

\section{El miedo como principal consejero político tras el 11-S}

En el nuevo milenio "la seguridad" se ha convertido realmente en una cuestión política de primer orden. Los principales partidos políticos han intentado desbancarse los unos a los otros realizando todo tipo de planes tan revolucionarios como poco realistas con los que convertir las calles en lugares más seguros. La seguridad se define cada vez más en términos negativos. Ya no está conectada con ideales positivos como la justicia social, la igualdad de oportunidades o la lucha contra las privaciones y la exclusión social. Desde ahora se interpreta principalmente como la forma de librar a las calles de la presencia de todos aquellos que amenazan la sensación pública general de seguridad. Junto a quienes de hecho roban o amenazan a los ciudadanos que observan las leyes, involucra también a vagos, mendigos, prostitutas de la calle, y a los llamados "adictos que rehuyen la asistencia". Ahora podemos hablar en Holanda de un revanchismo urbano emergente, del tipo del que hasta hace poco, sólo conocíamos en los Estados Unidos. La idea implícita es que los pobres de las ciudades han perdido su derecho sobre la ciudad porque son responsables de los delitos y porque la dilapidación y la desorganización parecen haberse convertido en algo generalizado (Uitermark \& Duyvendak, 2004).

La policía interpreta la nueva política de seguridad ciudadana como una carta blanca para reestablecer el orden público con métodos llamados "no ortodoxos". La expresión "no ortodoxos" es utilizada frecuentemente por la policía cuando propone prácticas de búsqueda y captura preventivas en algunos vecindarios deprimidos o cosas salvajes como colocar las fotografías de delincuentes habituales (la mayoría juveniles) en internet o meter a los rateros durante un tiempo en jaulas que sean visibles para los clientes de las tiendas en las que han hurtado. Los más salvajes de esos planes no pretenden ser puestos en práctica. Sirven para "sacudir" el debate público, según dicen los jefes de policia. A pesar de que todas estas prácticas tienen lugar en el ámbito local, no es muy distinto si "los medios no ortodoxos" para gestionar el delito y las molestias, son defendidos por políticos nacionales, autoridades locales o incluso por jefes de policía. Resulta plausible sostener que las diferencias locales se han tornado tan pequeñas debido a que se orientan contra un mismo enemigo: nuestra supuesta "tolerancia del pasado". Además, muchos municipios, "se emborrachan" bebiendo de la misma fuente. Visitando en masa a la Nueva York de Rudoph Giuliani y Bill Bratton, 
todos ellos han contribuido a lo que probablemente haya sido la mayor "transferencia de políticas" en la historia de la criminología convirtiendo "las ventanas rotas" y "la tolerancia cero" en verdades de Perogrullo primordiales para la política de seguridad local. La "tolerancia cero" se extendió por Holanda de un modo muy similar al que Trevor Jones y Tim Newburn (2004:132) nos explican con referencia al caso británico. Esto es, "no tanto en el campo de la acción policial como en el más genérico de la retórica y el discurso". Como en el caso británico, las preocupaciones clave de las actividades policiales nuevayorquinas de tolerancia cero -elevado índice de homicidios, oleada de vandalismo urbano o mendicidad ${ }^{4}$ agresiva- no eran temas importantes en Holanda. No obstante, uno de los efectos indeseables de las prácticas policiales de la tolerancia cero de Nueva York -el aumento de la criminalización y la estigmatización de las minorías étnicas- se hace notar en el caso holandés.

Aunque desde que Holanda es un país de recepción de inmigrantes siempre ha habido periodos breves con algunas tensiones raciales, resulta un fenómeno relativamente nuevo que prácticamente todos los problemas sociales sean juzgados a partir de parámetros étnicos. Ahora parece como si de repente todo se hubiese transformado en una cuestión de "nosotros" ciudadanos holandeses blancos contra "ellos", los inmigrantes (en especial aquellos de procedencia musulmana) que rechazan adaptarse a las normas de "nuestra" ilustrada civilización occidental. Se ha convertido prácticamente en una excepción encontrar en los periódicos artículos sobre molestias, bandas juveniles, ajustes de cuentas o terrorismo que no haga referencia al origen étnico o cultural de los sospechosos. Esta sobre estigmatización, y el hecho de que se salga fácilmente con cualquier comentario racista son fenómenos nuevos.

Debe quedar claro que una preocupación genuina y profunda por la experiencia general ante modales groseros y por un aparente y permanente alto nivel de delincuencia juega un importante papel en el debate público actual sobre seguridad. Con todo, de inmediato debe también decirse que existen escasas pruebas empíricas en apoyo de la idea de una crisis normativa (WRR, 2003). Aunque siempre debemos ser muy cuidadosos con los índices oficiales de delincuencia y con las estadísticas sobre riesgo de delitos, podemos decir con seguridad que están decreciendo desde ya hace algunos años. Sin embargo, al mismo tiempo también es verdad que una gran parte del comportamiento antisocial que encontramos en las calles involucra a jóvenes de minorías étnicas de los que se dice que son, además, bastante más refractarios que los jóvenes blancos holandeses. También las formas más extremas de violencia en el ámbito público (en especial tiroteos en las escuelas, restaurantes, centros comerciales o parques) tienen en su mayoría una dimensión étnica. Y esto es aquello que la gente ve y frente a lo que reacciona. Pero para comprender plenamente la enorme preocupación por la seguridad y la casi culpabilización axiomática de los musulmanes "inadaptados" por todo lo que sale mal, tenemos que mencionar tres fechas concretas: el 11 de septiembre de 2001, el 6 de mayo de 2002 y el 2 de noviembre de 2004.

Excepto Gran Bretaña, no existe probablemente otro país europeo que se identifique tan fuertemente con los Estados Unidos como Holanda. A pesar de todas las críticas, el tono general todavía recuerda que los estadounidenses nos liberaron de los

\footnotetext{
${ }^{4}$ En inglés "panhandling". "Panhandling," es un término común en Estados Unidos, aunque con más frecuencia se use "begging", y en alguna ocasión "cadging." El término que nos ocupa se refiere en general, a vagabundos, mendigos, gorrones, pordioseros y supone un caso de metonimia puesto que evoca la imagen de quien, después de usar el platillo o cazueleta para la extracción de oro en las minas (pan) del Oeste Americano, lo usó para pedir limosna extendiendo el brazo con dicho platillo en la mano. Se considera agresiva la mendicidad en la que se acosa al transeúnte en grados diversos para conseguir mediante alguna intimidación la "limosna". (N. de la tr.)
}

Revista Española de Investigación Criminólogica

REIC AC-02-05

http://www.criminologia.net

ISSN 1696-9219 
nazis y que, por tanto, nuestra obligación es estar del lado de los Estados Unidos. Así los ataques al las Torres Gemelas de Nueva York y al Pentágono se interpretaron como un ataque contra nosostros. Desde el "11-S" existe una sospecha generalizada sobre todos los musulmanes de ser terroristas potenciales que también pueden atacarnos, a pesar de que nuestro país los haya tolerado (isic!) como visitantes durante muchos años. Las mezquitas -y en particular quienes las financian, frecuentemente saudíes- se encuentran sometidas a vigilancia permanente por el servicio secreto holandés $\mathrm{AIVD}^{5}, \mathrm{y}$ se han seguido desde entonces numerosas detenciones de islamistas radicales. Desde el "11-S" gran parte de la población musulmana dice sentirse acorralada y amenazada por la sospecha. Un magistrado del Tribunal Supremo se ha manifestado abiertamente en contra de las propuestas de ley antiterrorista, ya que pondrían al estado de derecho en estado de sitio (Corstens, 2005). ${ }^{6}$

La segunda fecha se refiere a un evento nacional: el asesinato del popular líder político de derechas Pim Fortuyn. Como secuelas de estos dos acontecimientos, emergió un nacionalismo insoportable y estrecho de miras, en el que la "seguridad" adquirió una connotación negativa. Cuando el partido de Pim Fortuyn (el LPF) sostenía en 2002 que era el partido de la Ley y el Orden, introdujo el término habitabilidad (leefbaarheid en holandés) como un rumor en la agenda política. Mientras que casi todos pensaban que las cosas iban bastante bien, Fortuyn sugirió de repente que los gobiernos anteriores habían hecho de Holanda un lugar inhabitable y lo habían llevado al desorden. Las listas de espera de la asistencia médica, los embotellamientos de tráfico, el caos en el sistema educativo y una generalización de los incidentes llamados "de violencia sinsentido" eran la prueba de ello. El asesinato de Fortuyn dio a la cuestión de la seguridad pública y de la habitabilidad un papel todavía más prominente en la agenda. El mismo hecho de que un político "que por fin se atrevía a decir lo que la gente en general pensaba" fuese asesinado, fue la última prueba de que algo iba yendo realmente mal en este país. También probó para muchos ciudadanos corrientes que los "políticos anticuados" no tenían ni idea de cuan insegura se había tornado nuestra sociedad". Ellos fueron acusados, junto con la "élite liberal" de los que se decía que dominaban los medios de comunicación y el sistema educativo, de crear el clima en el que tal asesinato podía tener lugar. Es alarmante que debido principalmente a dicho incidente el populista partido de derechas fuese capaz de convertirse en las elecciones nacionales de 2002 en el segundo partido más votado del país con tan solo una agenda muy general sobre el descontento y sin ninguna propuesta de solución ${ }^{8}$.

\footnotetext{
5 Hasta el 29 de mayo de 2002 el servicio secreto holandés había sido el BVD (Binnenlandse Veiligheidsdients) o Servicio de Seguridad Nacional, pero la limitación que el término "Nacional" imprimía a su nomenclatura fue descartada entonces, pasando a ser el AIVD (Algemene Inlichtingen en Veiligheidsdients) o Servicio General de Inteligencia y Seguridad. (N. de la tr.)

${ }^{6}$ El titular, en esta intervención tan poco común del poder judicial en el debate parlamentario sobre el terrorismo, que aparecía en el rotativo líder nacional NRC Handelsblad (12 y 13 febrero de 2005, pp.3334) era, a este respecto, bastante ilustrativo: "Culpable, hasta que no se demuestre lo contrario".

7 Se ha sostenido con frecuencia que el último asesinato político en Holanda fue el de Guillermo de Orange en 1584 a manos de un sicario contratado por el opresor español del país por aquellas fechas. Pero esto no es cierto; ha habido muchos asesinatos políticos en la historia de Holanda, el último tuvo probablemente lugar en la guerra de independecia indonesia (1945-49). También en un contexto europeo los asesinatos políticos no son una excepción; mencionaremos aquí el asesinato de André Cools en la vecina Bélgica en 1991, a instancias de los miembros de su propio partido político.

${ }^{8}$ El LPF (Lijst Pim Fortuyn) se fundó en gran medida por la industria de la construcción e integra a todo tipo de "nuevos ricos", bárbaros, neoconservadores que fueron comunistas de jóvenes, antiguos ultra conservadores revanchistas que perdieron el tren cuando eran jóvenes, tradicionalistas decepcionados, y la clase trabajadora votante de la izquierda que se sintió abandonada por su propio partido. Vee: van Swaaningen, 2003.
}

Revista Española de Investigación Criminólogica

REIC AC-02-05

http://www.criminologia.net

ISSN 1696-9219 
Cuando Fortuyn fue asesinado, muchas personas progresistas estuvieron deseando que el asesino no fuese musulmán. Resultó ser un holandés blanco ecologista radical. Este hecho desembocó en una condena general de la izquierda que, demonizando a Fortuyn, había creado el ambiente propicio para que tuviese lugar el asesinato. Dicha acusación dejó durante bastante tiempo a la izquierda en una situación de impotencia. El 2 de noviembre de 2004 tuvo lugar en Holanda otro asesinato político: un joven holandés de origen marroquí, islamista radical, asesinó al cineasta Theo van Gogh, quien había insultado a la comunidad musulmana a través de una columna de diario especialmente ofensiva y en un documental sobre los abusos sufridos por las mujeres en las comunidades musulmanas ${ }^{9}$. A resultas de tal situación, se incendiaron mezquitas y escuelas islámicas, se produjeron detenciones de radicales islamistas (nacidos en Holanda y muy jóvenes) y dos miembros del Parlamento, con agendas anti Islam, fueron, tras duras amenazas, forzados a esconderse. Todo ello, fomentó más si cabe las tensiones étnicas. Tras el asesinato de Theo van Gogh las comunidades musulmanas como un todo fueron responsabilizadas bajo la misma confusa racionalidad con la que se acusó a la izquierda a la muerte de Fortuyn.

A propósito de dicho extremo, resulta interesante tener en cuenta que este tipo de represalias sobre la comunidad musulmana permaneció ausente de la escena española tras los atentados del 11 de marzo de 2002, y que la izquierda no fue estigmatizada. De hecho ocurrió lo contrario: el gobierno conservador de Aznar fue mandado a casa porque mintió sobre lo ocurrido en la estación de Atocha, y el partido socialista alcanzó el gobierno tras las elecciones. Probablemente la diferencia haya venido causada por el hecho de que los ciudadanos españoles han sufrido la violencia "terrorista" durante largo tiempo, mientras que para los holandeses eso es algo que no encaja en la imagen que la gente tiene de su país. Además, en 2004 el populismo ya ha permeado de tal forma la política y los medios de comunicación que políticos y periodistas más bien se dedican a "traficar" con el miedo que a colocarlo en perspectiva (Mak, 2005: p.38).

En la era de la europeización política y de la globalización económica parece que el horizonte mental de los holandeses no alcance a ir más allá de la vuelta de la esquina. Hoy por hoy el término "comunidad" se utiliza de un modo estrecho y nostálgico. El mensaje implícito es el siguiente: nuestras comunidades estaban seguras en el mundo preglobalizado de los años cincuenta, en el que la "gente corriente" todavía podía entender el mundo circundante, por lo tanto debemos volver a esa situación. La candidez política implícita en este mensaje es, por lo demás, bastante increíble. Como país de comerciantes hemos abrazado la política neoliberal de la globalización -y así desbaratado muchas economías locales de pequeña escala- pero no nos esperábamos "que los malos de ahí afuera" desbaratasen nuestro pacífico país. Hemos apoyado los bombardeos y ocupaciones estadounidenses de los países musulmanes, pero estamos sorprendidos de que los musulmanes nos culpabilicen por ello.

El que una vez fue el ideal multicultural había terminado, se dijo, en el drama multicultura $^{10}$. Ha llegado el tiempo de que los forasteros se adapten a nuestras normas

\footnotetext{
${ }^{9}$ Van Gogh se refirió duramente a los musulmanes como "a la quinta columna de jodidos cabrones". Sin embargo en sus filmaciones fue más sutil. Por ejemplo realizó unas bastante más sutiles series televisivas sobre la historia de amor multicultural de "Najib y Julia". Su última película se basaba en un guión de Ayaan Hirsi Ali, un parlamentario holandés de procedencia somalí, y retrataba la paliza de una mujer con chador transparente que tenía escritos en su cuerpo textos del Corán que perdonaban los abusos sobre mujeres.

10 La expresión fue lanzada por el periodista Paul Scheffer en un artículo del diario NRC Handelsblad publicado el 29 de enero de 2000. Es digno de atención que Scheffer, precisamente como Fortuyn, fuese un marxista en los años setenta. Un alto número de excomunistas holandeses dieron un enorme giro a la
}

Revista Española de Investigación Criminólogica

REIC AC-02-05

http://www.criminologia.net

ISSN 1696-9219 
y a nuestros valores - cuales sean queda en suspenso - o dejen el país. En la era de la europeización política y de la globalización económica parece como si de repente nuestro horizonte moral no llegase más allá de la vuelta de la esquina. Ahora mismo Holanda parece haber abrazado el terror, el resentimiento y el enfado y los políticos con frecuencia actúan con un pánico increíble de una forma bastante irreflexiva e inhumana.

\section{Una política de seguridad ciudadana guiada por la brújula del miedo}

El movimiento gradual desde la prevención del delito, vía la seguridad ciudadana hacia la seguridad pública y la "habitabilidad" constituye un desarrollo bastante lógico con la línea de política jurídico criminal iniciada a mitad de los años ochenta. Excepto por algunos distintos acentos de matiz, fue adoptada por los gobiernos cristiano demócratas, laboristas y conservadores. Todos esos partidos fueron descartados como "políticos anticuados". Después de todo "los políticos anticuados" representaban la tecnocracia y la ausencia de receptividad. Los políticos anticuados hablan un lenguaje que nadie excepto los de su propia casta administrativa autocomplaciente entiende. Incluso aunque el partido de Pim Fortuyn (LPF) volvió a ser la incompetente flor de un día, la base social desde la que emergió ha causado un giro más permanente hacia la derecha y un estilo de debate más populista, tanto en los medios como entre los políticos ${ }^{11}$.

En estos días, los proyectos de seguridad pública de las principales ciudades holandesas se centran más y más en la lucha contra el delito en las calles. Puesto que Rotterdam es la fortaleza de la derecha populista en Holanda, vale la pena detenerse a ver qué es lo que allí está ocurriendo. El proyecto de seguridad pública de Rotterdam habla explícitamente de la lucha contra el delito y contra otras conductas incívicas. Sus términos clave son "molestias", "limpio y reparado" (leído en un contexto de "ventanas rotas") y "tolerancia cero". Los blancos de ataque clave son los llamados delincuentes juveniles habituales, los "inmigrantes delincuentes ilegales", los "adictos que rehuyen la asistencia" y las prostitutas de la calle (Gemeente Rotterdam, 2002 a\&b). Pero también en la supuestamente tolerante ciudad de Ámsterdam la policía ha comenzado a castigar conductas meramente incívicas como montar en bicicleta sin faros, $\mathrm{u}$ orinar en un árbol o en una esquina - acciones que habían sido consentidas durante las pasadas décadas. Estas nuevas prioridades tienen que ser muestra de que la policía se toma en serio las sensaciones de los ciudadanos que observan las leyes. Con ello el delito se ha convertido en un problema de orden público. Cuando la inseguridad subjetiva se erige en brújula de las políticas de seguridad públicas, las prioridades de la policía cambian y llegan a incluir la lucha contra conductas no delictivas que ahora son llamadas "comportamiento criminógeno" o "molestias".

La penalización de las molestias se justifica con argumentos preventivos. Se sostiene que tolerar o aceptar dicha mala conducta empeorará los problemas. Los delincuentes del futuro anuncian su llegada causando molestias, haciendo campana y

derecha en los noventa. En Holanda el primero en señalar los límites del multiculturalismo -en un modo bastante más basado en cuestiones socioeconómicas- fue el partido socialista radical. Este fue entonces acusado por el resto de partidos, incluido el conservador VVD y CDA, quienes son hoy los más fieros defensores de un enfoque "más severo" frente a las minorías étnicas. Los más fuertes protagonistas del multiculturalismo, los laboristas y los verdes, guardan ahora silencio sobre este tema.

${ }^{11}$ El término "populismo" se utiliza a menudo para desacreditar a ciertas personas o movimientos. Aquí lo tomo en su sentido más literal; esto es, como un rechazo de los valores de las élites o del establisment y una actitud escéptica y de sospecha frente a los expertos. El populismo frecuentemente apela al "sentido común" para que el discurso académico o intelectual parezca trivial y confía en las opiniones de la "gente corriente" ( $c f$. Elchardus, 2002:90)

Revista Española de Investigación Criminólogica

REIC AC-02-05

http://www.criminologia.net

ISSN 1696-9219 
orinándose en las calles. La prohibición de la mendicidad en Rotterdam, mencionada en la introducción, tiene que ser entendida en este contexto. Se presenta un interesante dilema cuando una cierta medida tiene efectos distintos sobre la seguridad "objetiva"y sobre la subjetiva. ¿Cómo tiene que comportarse la autoridad local si la investigación demuestra que la instalación de cámaras de circuito cerrado en determinada zona no reduce el índice de delitos, pero mejora las sensaciones de seguridad de la gente? ¿Debería invertirse en un placebo? Si la respuesta es afirmativa ¿En qué medida debería hacerse? ¿Sería suficiente por ejemplo con colocar cámaras falsas, que cuestan menos de un décima parte de lo que cuestan las que funcionan de verdad y cuyas cintas tienen que ser visionadas por la policía?, o por el contrario ¿Sería mejor invertir el dinero en oficiales de policía para que efectivamente patrullen las calles?

Aunque particularmente los recientes proyectos políticos de la ciudad de Rotterdam sugieren una enorme resolución, su interpretación e implementación real permanecen inciertas. "Dejar las calles limpias" suena muy contundente, pero no está claro qué ocurre después con todos esos jovencitos, adictos, prostitutas, mendigos, extranjeros "ilegales" que han sido "quitados de en medio". Sólo tienen que apartarse de nuestra vista. Son llevados a lugares en lo que son menos molestos, enviándolos a prisión, intentando deportarles a los países a los que las autoridades creen que pertenecen (lo que muchas veces no es fácil de determinar) o incluso a zonas en las que pueden hacer menos daño. Esta parece ser la tendencia más importante en el control penal de nuestros días. Esta tendencia puede caracterizarse como un "destierro" al estilo moderno. El destierro es una exclusión temporal o permanente del delincuente o del potencial delincuente de ciertos lugares o funciones, y eso es exactamente lo que está pasando. Con la palabra "destierro", no me refiero a ninguna medida específica, sino más bien a la racionalidad utilizada para legitimar ciertas medidas. Déjenme ofrecerles algunos ejemplos.

El último Libro Blanco del Ministerio de Justicia holandés Hacia una sociedad más segura, anuncia una medida nueva para encarcelar a los delincuentes (juveniles) habituales por un periodo mayor de lo que el delito por el que han sido de hecho condenados justificaría normalmente. Así, son castigados por lo que son antes que por lo que han hecho. La nueva medida no pretende influir en la conducta de los presos, sino que propone un encarcelamiento sin mayores ilusiones; i.e. un destierro de la sociedad por cierto periodo de tiempo ${ }^{12}$.

También en la esfera del crimen organizado podemos contemplar formas de destierro. La nueva ley llamada BIBOB permite a las autoridades de manera preventiva denegar a determinadas personas licencias o incluso el permiso de compra de fincas en determinadas zonas si se tienen sospechas de que el dinero proviene de prácticas ilegales.

En Ámsterdam la normativa local administrativa permite a la policía alejar a una persona para conseguir terminar con determinada práctica, sin realizar ningún arresto o dar inicio a proceso alguno. A esta práctica se la denomina "orden de arresto preventivo" (tegenhouden en holandés). Un ejemplo de tal práctica lo constituye la deportación de algunas mujeres nigerianas sospechosas de llevar a sus clientes fuera de las zonas legales de práctica de la prostitución y robarles. No se realizó ningún informe formal ni fue seguido ningún procedimiento: sencillamente se las subió al primer avión con destino a Nigeria.

Un nuevo ejemplo, muy diferente que ejemplifica dicha práctica: actualmente la policía investiga la posibilidad de utilizar moderna tecnología para el reconocimiento de

\footnotetext{
${ }^{12}$ Para un análisis más detallado de esta tendencia en el sistemas de prisiones véase: uit Beijerse \& van Swaaningen, 2003.
}

Revista Española de Investigación Criminólogica

REIC AC-02-05 $\quad \underline{\text { http://www.criminologia.net }}$

ISSN 1696-9219 
personas que han sido una molestia en ciertos lugares, para así denegarles el acceso a otros como el transporte público, las piscinas o los centros comerciales. En cierta medida esto ya se aplica en los estadios de fútbol. Tan sólo estamos esperando el momento en que los sistemas de identificación estén conectados entre sí para crear una base de datos en la que todos los que tengan algún tipo de "orden de destierro" sean registrados...

Viendo todas estas formas de "prevención del delito" al nuevo estilo, podría pensarse que cada uno de los proyectos originales de seguridad ciudadana está muerto y enterrado, pero ese no es el caso. Resulta bastante significativo que nadie haya dicho jamás que estos eran un error y que por ello se tuviesen que tomar nuevos derroteros. Justus Uitermark and Jan Willem Duyvendak (2004) ofrecen una explicación muy práctica de por qué las autoridades locales han continuado desarrollando políticas sociales a pesar de la dureza de su retórica. En primer lugar, las autoridades no pueden abandonar por completo el sendero de sus predecesores, ya que las políticas se desarrollan en un contexto dado y es necesario adaptarse a ese contexto con objeto de ser efectivos. En segundo lugar, las ideas de los funcionarios públicos que tienen que desarrollar esos nuevos programas no cambian de la noche a la mañana. A pesar del giro radical en el discurso sobre política de seguridad, la práctica se muestra de hecho bastante más continuista.

Pero... parece que algo sí ha cambiado. Es cierto que palabras como "vecindario" o "comunidad" son utilizadas con profusión en recientes documentos políticos, pero en la práctica se observa de facto una tendencia muy diferente: tanto los políticos como la policía se apartan de las ideas de "construcción de la comunidad" y de la integración. Algunos de los que en su momento fueron celebrados centros de justicia vecinales $(\mathrm{JiB})$ han sido ya cerrados alegando "razones de eficiencia", y a los que permanecen abiertos se les ha asignado la tarea de disminuir el índice de delitos para la fiscalía. Se supone que en el vecindario ya no se tiene que invertir en relaciones "multi institución". Las patrullas ciudadanas también parecen haber pasado de moda. La policía ahora se ve a sí misma como "guardianes" y se centra en "desterrar" de ciertos vecindarios a los jóvenes problemáticos. En palabras de Jock Young (1999) podemos observar una clara tendencia al pasaje de una sociedad "incluyente" a una "excluyente".

\section{Tomarse los miedos en serio}

El común - si bien incorrecto - argumento consiste en decir que las nuevas políticas de la Ley y el Orden resultan una reacción a la desubicada permisividad del pasado, al aumento de la tasa de delitos y al ingente aumento del flujo de extranjeros. "Desafortunadamente" (para este argumento) la tasa de delitos en su conjunto no ha aumentado durante las última dos décadas, el flujo de extranjeros de hecho ha decrecido e incluso argüiría que los efectos de la llamada "desubicada permisividad del pasado" han sido exagerados groseramente. Probablemente la razón por la que se mantiene dicha visión contra todo pronóstico tiene mucho que ver con una profunda preocupación frente a las nuevas - afortunadamente todavía raras - formas de violencia muy seria en la que se ven involucrados con frecuencia los extranjeros. El sociólogo belga Mark Elchardus acusa a los criminólogos de haber restado importancia a tales sentimientos, excluyéndolos nosotros mismos del debate público sobre el delito. Aunque este argumento también se sostiene en Holanda, no estoy seguro de en qué medida de hecho afecta al caso holandés. El movimiento de "tomarse los miedos en serio" y enfrentar la cuestión étnica que tan a menudo se encuentra involucrada, tuvo lugar en Holanda 
bastante más pronto, digamos que en torno a los años noventa. ${ }^{13}$ En mi opinión lo estamos ahora llevando demasiado lejos, y con frecuencia contrarrestándolo muy poco en los medios de comunicación con las dramáticas historias de robos a marroquíes, apuñalamientos de antillanos o palizas a turcos y yugoslavos. No obstante, tomar en serio tales casos y la ansiedad que producen parece el punto más relevante que debe tenerse presente si queremos reivindicar un papel crítico en el debate público sobre el delito y el control penal. Sólo entonces podremos dar a conocer nuestros importantes puntos acerca de causas y posibles soluciones al respecto y posiblemente podamos desviar la "espiral de dramatización" de nuevo en la otra dirección.

Con respecto a este último problema el papel de los medios es el más obvio. Sabemos que los medios son un amplificador de los miedos y hoy en día esto es así en mayor medida que en los años setenta. El documental de Michael Moore Bowling for Columbine muestra adónde nos puede llevar esto: se crea a gente con miedo y la gente con miedo pide "medidas drásticas" como si fuesen espejitos mágicos que pueden llevarse sus miedos. Como tales son insaciables y ni siquiera las medidas más draconianas les resultan suficientes. Mark Elchardus (2002) llama a este estado en el que nos encontramos una "dramademocracia". En una "dramademocracia" la realidad y la ficción son casi indistinguibles. La "TV Reality" en cierta medida ha mutado en algo más real que nuestra propia realidad de cada día y las "estrellas" de los culebrones se han convertido en nuestros conciudadanos. De ser ciudadanos comprometidos preocupados por los problemas reales de la vida real, hemos pasado a ser espectadores de un culebrón - o de un show de horror si prefieren - sobre el delito y el control del delito. Esto hace más fácil de entender por qué ahora los "hechos" parecen tener un impacto relativamente menor en lo que la gente piensa y por qué las sensaciones juegan un papel tan importante en la política de estos días. Si esto es cierto ¿Qué es lo que nos queda a los académicos, cuya empresa radica en gran medida en separar la realidad de la ficción? Creo que debemos seguir repitiendo los obvios hechos empíricos sobre el delito y el control penal. La avalancha de pánicos morales y respuestas irreflexivas pueden habernos paralizado durante la última década. Sin embargo, las investigaciones muestran que las personas bien informadas realizan juicios distintos a los de los escasamente informados, con independencia de su nivel de educación o de sus preferencias políticas. Todavía quedan suficientes personas interesadas en los matices. Además esas personas generalmente siguen ocupando posiciones que pueden marcar la diferencia. También somos profesores, educando a una generación que probablemente ocupará tales posiciones en el futuro.

\section{El papel de la economía}

Relacionar las variaciones en la comisión de delitos y en el control penal con el desarrollo cultural y económico es uno de los modelos explicativos mas importantes en criminología desde la aparición de Deux lois de l'évolution pénal en 1902 de Durkheim o de Punishment and Social Structure en 1939 de Rusche y Kirchheimer. Sin embargo, la crisis económica y el aumento de la punitividad no han ido de la mano en el caso holandés (de Haan, 1990: capítulo 3). Con la crisis económica de 1973, las tasas de encarcelamiento continuaron descendiendo, y después de la crisis económica de principios de los ochenta solo aumentaron escasamente. El mayor aumento del sistema de prisiones y la explosión contemporánea de sanciones administrativas tuvo lugar a

13 Y la noción más amplia de "tomarse los delitos en serio" fue acuñada al inicio de los años ochenta, justo después de que los realistas de la izquierda británica introdujesen la cuestión en la agenda criminológica.

Revista Española de Investigación Criminólogica

REIC AC-02-05

http://www.criminologia.net

ISSN 1696-9219 
finales de los años ochenta y en los noventa, cuando la economía era floreciente. En parte por ello, parece que vale la pena investigar más detenidamente la posible relación entre el aumento de la punitividad y la polarización socioeconómica. Tradicionalmente Holanda ha sido una sociedad con un buen nivel de vida, pero desde los años ochenta hemos visto un fuerte incremento del número de pobres $y$ del número de Porsches. Las personas en una situación difícil tienden a buscar chivos expiatorios a los que culpar de sus desgracias. En este sentido hay que destacar, que las extremadamente generosas recompensas y los premios que se han vuelto normales en el mundo de los negocios también se viven como un ejemplo de crisis normativa al modo de la de los modales groseros en las calles ${ }^{14}$. Otro hecho -que no encaja fácilmente en este análisis- es que tanto los chivos expiatorios como los protagonistas más orgullosos de la Ley y el Orden, provienen de los grupos de renta más baja.

Storm y Naastepad (2003) sostienen que la globalización neoliberal de los años noventa ha contribuido enormemente en la "angustia holandesa" de la que la actual preocupación por la seguridad es un claro exponente. Señalan a tres "modos de huir" de la sensación de inseguridad económica y social que mucha gente corriente percibe hoy en día. Cuando el "sentido del yo" se encuentra altamente determinado por el éxito del mercado, mucha "gente corriente" pierde confianza en sí misma. Robert Merton ya hace tiempo que señaló las tensiones que "el sueño americano" había causado, pero este es un fenómeno bastante nuevo en la tradición social democrática holandesa. La primera huida la proporciona el propio mercado: i.e. mediante una forma competitiva de consumismo. La segunda huida la ofrece el chovinismo: i.e. subrayando que "nosotros" tenemos nuestro propio orgullo nacional y que este nada tiene que ver con las fuerzas del mercado. La tercera, por último, consiste en "la glorificación del éxito competitivo" entre las clases sociales desfavorecidas. Según Storm and Naastepad, Fortuyn fue uno de los pocos políticos que entendió las paradójicas reacciones a la globalización neoliberal y fue capaz de acomodar las tres en su agenda política. Este análisis parece un camino fructífero que seguir si queremos comprender los determinantes socioeconómicos de la actual demanda de protección y seguridad.

\section{Una cultura de la firmeza y del "vitalismo"}

Una inseguridad existencial relacionada, que también forma parte del actual clima político de descontento y rencor, se halla en lo que se ha llamado "la emergencia de una cultura de la firmeza" en la que la élite bien parlante y bien educada determina cómo van las cosas. Una nueva categoría de "huérfanos rezagados", integrada por la tradicional clase trabajadora así como por hombres hechos a sí mismos extraordinariamente ricos, pero prácticamente autodidactas - se sienten ignorados, tan sólo por no expresar sus deseos y reclamos en una forma lo suficientemente equilibrada y corroborada y por decir "simplemente lo que sienten". Las privaciones, podría sostenerse, ya no son meramente una cuestión de dinero, sino primariamente de educación. Esto nos tiene que enseñar algo sobre nuestras teorías criminológicas de la privación y las subculturas. ¿Quienes son hoy los grupos vulnerables? ¿Son los chavales excluidos socialmente, desempleados (inmigrantes), sin educación o con muy poca, que aterrorizan por los alrededores con un estado de frustración, o son los igualmente tan necesitados económicamente, habitantes (blancos) del barrio que se sienten amenazados e intimidados por esos chavales?

\footnotetext{
${ }^{14}$ Según una encuesta sobre los cambios de opinión acerca del delito y de las penas, llevado a cabo por nuestro departamento, que fue publicado en el diario nacional Trouw, en la edición del 14 de enero de 2004, pp. 11-13.
}

Revista Española de Investigación Criminólogica

REIC AC-02-05

http://www.criminologia.net

ISSN 1696-9219 
Nils Christie (1993: 41-46) afirma que la tolerancia holandesa de hecho es una tolerancia desde arriba. Es la tolerancia de una élite liberal, y no la que apoya el gran público. En cierto sentido esta crítica parece injustificada. Por lo que concierne al comportamiento que en realidad no molesta a nadie parece de justicia decir que "los holandeses" todavía son personas bastante tolerantes y razonables. La cuestión es que la tolerancia frente a conductas más molestas es tan baja como el cualquier otro país y probablemente sea así entre la "gente corriente", sólo porque la élite puede permitirse ser tolerante ya que difícilmente tendrá que hacer frente a las consecuencias negativas de ese tipo de comportamiento. Sobre este particular las observaciones de Christie sin duda son correctas. En este momento la élite liberal ha perdido mucho terreno, y en su lugar tenemos populismo. El reto probablemente consista en demostrar que la retórica populista ofrece soluciones muy pobres, y que todavía tiene sentido realizar un análisis en profundidad antes de actuar y cumplir los importantes límites normativos del Estado de Derecho. Probablemente la élite liberal ha dado todas estas cosas por descontadas y no ha sabido explicarlas en términos convincentes para el gran público. Si usted piensa que vale la pena luchar por esto, cabe una defensa mucho mejor. Tal vez sea sólo cuestión de tiempo. Si la derecha populista continua más bien con la política socioeconómica anti social que está llevando a cabo, "la gente corriente" se dará cuenta de que no están hablando en su nombre. Puede ser que entonces reclamen a personas capaces, que se presenten con propuestas mejores a las que ellos mismos habían pensado.... también por lo que hace a abordar el delito. Debe además señalarse que la llamada élite liberal holandesa todavía maneja la mayoría de sistemas sociales. En la esfera de la justicia penal son los políticos, los medios de comunicación y la policía quienes juegan la carta populista, pero ésta es menos visible en el sistema penitenciario, el poder judicial o las autoridades locales. También hay choques bastante interesantes entre autoridades nacionales y locales - en especial con respecto a la deportación de extranjeros "ilegales".

La actual cultura del control (Garland, 2001) a menudo se entiende relacionada con una presunta "falta de control" en las décadas precedentes. Según Gabriël van den Brink (2002), los años sesenta pueden ser entendidos como un impulso hacia mayor libertad, puesto que la era precedente se caracterizó por muchas restricciones. Un enfoque tolerante frente a formas, según se afirmaba, inocentes de desviación era un exponente de este deseo de libertad -en particular de la libertad del liberal cultivado de clase media, añade Gabriël van den Brink. En los años ochenta, corrimos contra los límites de esa libertad, porque había hecho descarrilar en la más pura indiferencia frente a todo tipo de comportamiento dañino - del que principalmente las clases obreras tradicionales eran las víctimas. Con su emancipación presunta alrededor del cambio de milenio, surgió un contramovimiento. La ideología de la libertad absoluta es ahora sustituida por un deseo de nuevas restricciones ${ }^{15}$. Según van den Brink a la élite liberal le llevó hasta 2002 reconocer la cultura de descontento que había permanecido latente durante ya aproximadamente siete años. Incluso aun cuando podamos tener nuestras dudas sobre la exactitud de este análisis, en él resuena el importante cambio político que ha sido provocado por Pim Fortuyn. Después de que las autoridades fuesen criticadas por imponer sus posiciones moralistas en los años 1960, el ciudadano medio ahora espera que el Estado dicte normas claras y castigue a aquellos que las violan. La gente

\footnotetext{
${ }^{15}$ Excepto por el prefacio, van den Brink no es tan explícito sobre esto en el libro; mi paráfrasis se basa más en una entrevista ofrecida para el lanzamiento del libro en el NRC Handelsblad, 28/29 de diciembre de 2002.
}

Revista Española de Investigación Criminólogica

REIC AC-02-05 $\quad \underline{\text { http://www.criminologia.net }}$

ISSN 1696-9219 
suele pedirle mucho al Estado, y ve de manera muy limitada su papel personal (por ejemplo con respecto al fortalecimiento del control social o de las normas sociales) ${ }^{16}$.

Esta tesis también hace surgir nuevas preguntas sobre los motivos por los que estos ciudadanos corrientes de repente se "revolvieron" contra la élite liberal. Parece bastante tentador volver a leer a todos los clásicos que tratan sobre la aparición de los movimientos de masa, en particular aquellos sobre la psicología de masas en el fascismo ${ }^{17}$. En la situación holandesa la actual "rebelión de las masas" se explica por lo general señalando las incertidumbres existenciales de la "gente corriente" en la nueva sociedad multicultural globalizada. Se sostiene que se reacciona de un modo tan a la defensiva y xenófobo porque ya no se entiende ni lo que pasa en la propia comunidad tanto menos en su ciudad o su país, o en Europa (que nunca ha sido suya) o en el mundo como un todo. ¿Esto es simplemente la nostalgia de los ancianos sobre los "viejos buenos tiempos" combinada con la visión de los jóvenes, más bien poco comprometidos políticamente, consentidos, hedonistas y "vitalistas" que quieren sacarse de encima todo lo que no sea divertido y guay o hay en juego algo más fundamental?

Hans Boutellier en su reciente libro La Utopia de Seguridad describe el aumento de la atención política (y mediática) prestada a la seguridad primariamente como una consecuencia del aumento de sensibilidad frente al delito y la victimización. Sin embargo, de hecho, dibuja unos parámetros que van mucho más allá de eso. Boutellier explica el aumento de la preocupación por la seguridad como una consecuencia de nuestra cultura postmoderna del riesgo y como una tensión psicológica fundamental entre nuestro vitalista reclamo de libertad y nuestro deseo de ser protegidos contra las consecuencias de la libertad de otras personas (Boutellier, 2004). El concepto de "vitalismo" es bastante interesante, puesto que dice algo sobre un impulso cultural general detrás del delito, en lugar de centrarse en ciertos "grupos de riesgo" y su control como predominantemente se hace en el debate sobre la seguridad ciudadana. El vitalismo incluye la vida rápida de fiestas salvajes, píldoras y emociones. La gente joven en particular parece "necesitar" una vida llena de "acción” y se aburre fácilmente. Esto hace a nuestra sociedad, por una parte, más dinámica e innovadora que antes pero, por otra, conforma también un impulso importante detrás del "carnaval del delito" (Presdee, 2000).

Así, siguiendo de nuevo a Boutellier, "el vitalismo" es simultáneamente el punto más fuerte y el punto más débil de nuestra sociedad. Ahora, ese problema es obviamente un poco más grande que el mero delito. Esto también nos lleva a la conclusión de que nuestros altos índices de delitos son hasta cierto punto inevitables, y con ello, a la idea de que el deseo general de más control tendrá escaso efecto al respecto. Pienso que deberíamos decir sólo esto, si bien Boutellier no parece compartir esta conclusión. Si nos fijamos en su estilo duramente crítico y peyorativo sobre la cultura juvenil, parece estar del lado de quienes continuamente se lamentan sobre la presunta falta de normas y valores, pidiéndole al estado que asuma un papel moral estrangulante. No obstante, al mismo tiempo, parece no creer en su propia receta.

Existen bastantes paradojas y puntos débiles a lo largo de todo su provocador libro; y ello es así, por lo siguiente: da por sentada la idea de que las culturas juveniles de hoy en día serían "vitalistas", sin señalar que los jóvenes también son caracterizados como poco comprometidos, pasivos alcachofados en el sofá que sólo miran estúpidos

\footnotetext{
${ }^{16}$ Según la misma encuesta del Trouw antes citada -nota 14-.

${ }^{17} \mathrm{Si}$ uno va a hacer esta compleja vinculación debería hacerlo bien. La cuestión es subrayar mecanismos similares, sin sugerir que el surgimiento del fascismo sería del mismo orden que la subida del populismo de derechas de nuestros tiempos. Ya que soy incapaz de hacer esto en este artículo, lo dejaré aquí como una sugerencia que necesita ser elaborada.
}

Revista Española de Investigación Criminólogica

REIC AC-02-05 http://www.criminologia.net

ISSN 1696-9219 
seriales, series de animación o programas musicales en televisión o juegan a marcianitos. Ambos retratos no pueden ser verdaderos simultáneamente. Sin embargo, el punto más débil del libro es que resta importancia a las tremendas consecuencias que para las libertades civiles, cuando sostiene que tenemos que prescindir de "alguna" libertad con objeto de ganar algo más de seguridad. Especialmente a la luz del debate sobre las -que están yendo muy lejos- nuevas medidas antiterroristas su tesis realmente necesita de una reflexión más seria. Un último elemento importante que debe mencionarse aquí es que el análisis de Boutellier pasa por alto uno de los elementos centrales del actual debate sobre seguridad en Holanda: la historia de lo que hemos llegado a conocer como el "drama multicultural".

\section{La incertidumbre en la sociedad multicultural}

En el siglo pasado, el multiculturalismo fue visto generalmente como el ideal para un país de inmigrantes como lo era Holanda. En torno al cambio de milenio, el ideal, se dijo, parecía haberse convertido en un drama. De manera retrospectiva se sostuvo que Holanda habría tenido una mala política de inmigración y de integración desde el influjo de las familias de los primeros "trabajadores invitados" a finales de los sesenta. Estas personas fueron admitidas de buen corazón, pero prácticamente no se esperaba nada de ellos. En ningún modo se les ofreció estímulos para que aprendiesen la lengua o para integrarse en la sociedad holandesa. En consecuencia, casi tenían que permanecer confinadas en el seno de su propia comunidad étnica. Sin embargo, la idea general es que el fracaso de su integración no se debió a que los holandeses no les quisieran, sino a los mismos inmigrantes, quienes habrían rechazado participar en la sociedad holandesa, que además era tratada con sospecha. Cualquiera que indicase problemas de adaptación de parte de los inmigrantes fue tratado durante mucho tiempo como racista. Se decía que esa actitud no encajaba en la auto imagen nacional de tolerancia de la élite liberal, y que "por lo tanto" mucha "gente corriente" guardó silencia.

En 2002, el carismático Pim Fortuyn de repente habló claro contra "los mimos" dispensados a las minorías étnicas y contra la naturaleza "atrasada", sexista y homo fóbica del Islam. Con ello fue a dar con una importante contradicción de la izquierda en Holanda en la que no se había reparado: la tensión entre la política del multiculturalismo y la política de la emancipación. Cabe resaltar que algunos de los miembros más emancipados de varias comunidades étnicas también se sintiesen atraídos por el mensaje de Fortuyn. Ellos mismos habían experimentado cuan mortificantes podían llegar a ser las cadenas étnicas y cómo éstas les habían impedido una participación significativa en la sociedad holandesa. ${ }^{18} \mathrm{Y}$, principalmente porque los poderes más conservadores del pilar islámico recibieron el apoyo del gobierno, apenas hubo renovación en el seno de las diferentes comunidades. Las parejas matrimoniales todavía son mayoritariamente buscadas (cuando no apañadas) en el país de origen y los niños que nacen y crecen en Holanda con frecuencia hablan un holandés muy pobre. Los que son considerados "demasiado holandeses" a menudo tienen por ello que romper con sus familias y con su comunidad, y los que son considerados "muy poco holandeses" no consiguen escalar posiciones sociales y están condenados a permanecer en puestos marginales. Hoy en día los periódicos van repletos de artículos sobre la integración y un comité parlamentario ha investigado recientemente la política de inmigración holandesa tras la guerra. Sus

\footnotetext{
${ }^{18}$ Portavoces clave de este nuevo partido político son de origen cabo verdiano, indostanés y marroquí. Otro punto interesante es que la agenda ultraconservadora de este partido obtenga un apoyo significativo de la comunidad gay - el mismo Fortuyn era abierta y flamígeramente gay.
}

Revista Española de Investigación Criminólogica

REIC AC-02-05 http://www.criminologia.net

ISSN 1696-9219 
conclusiones de hecho no son muy pesimistas ya que el comité sostiene que la integración de varias minorías étnicas en la sociedad holandesa con frecuencia ha tenido éxito a pesar de -antes que gracias a - la política del gobierno. ${ }^{19}$

El discurso de Fortuyn en términos de "o integrarse o marcharse" probablemente tuvo tanto eco, porque de hecho no cuestionaba la auto-imagen de tolerancia de los holandeses. Era el Islam el intolerante y el que bloqueaba la libertad de expresión. La relación entre los altos niveles de delitos en las calles y el multiculturalismo es por supuesto bastante más compleja de cómo la mayoría de las veces se describe, pero por otra parte sería muy fácil descartarla como si sólo fuese una obsesión racista. Simplemente existen problemas desproporcionadamente grandes con el delito en algunos grupos étnicos específicos. En tanto que criminólogos, podemos sostener que eso es bastante comprensible. Tan sólo baste señalar los enormes conflictos culturales entre "el mundo del hogar" y "el mundo de la escuela", el generalmente bajo nivel de educación, las altas tasas de desempleo, las pobres condiciones de alojamiento, y finalmente pero no por ello menos importante la exclusión y estigmatización social sobre la que también ha advertido el comité de investigación parlamentaria sobre la integración. Pero estas nociones escasamente llegan al gran público y, por lo demás, tampoco explican las enormes disparidades entre diferentes grupos étnicos que viven en condiciones socio-económicas parangonables. Por lo tanto, cualquier política de seguridad ciudadana está condenada al fracaso si la cuestión multicultural queda en el olvido...como así ha sido durante años. Inicialmente creo que tomar en consideración todo esto fue dar un paso adelante pero desafortunadamente ahora tiene todos los visos de ser un pánico moral.

\section{La jaula de hierro}

Un último tema en el discurso de la "habitabilidad" que deseo tratar aquí es el continuo proceso de ampliación y reorganización dentro del gobierno, la salud pública, la educación y otros servicios públicos que ha supuesto que estos se conviertan en impenetrables para la "gente corriente". Muchos de ellos simplemente veían a Pim Fortuyn como el Mesías que les liberaría del muro de formalismos y regulaciones que se habían ido acumulando. El partido social demócrata (PvdA) era visto como la encarnación de la tecnocracia, puesto que cada pregunta clara era contestada mediante parloteo administrativo sin sentido. Bajo su liderazgo, incluso las peticiones a favor de la desreguralización terminaban con la creación de más reglas y de más cuerpos de control. ¿Qué podemos aprender de esto en relación con el futuro de la seguridad ciudadana? Una importante frustración proviene de que las quejas, en una u otra forma, nunca parecen dirigirse a la persona correcta, ni presentarse en la forma debida, ni en el momento adecuado, y de que se tarde meses en recibir una respuesta y años para que de hecho pase algo. La burocracia en el seno de la policía, la administración de justicia y el gobierno local se ha convertido desde este punto de vista en la "jaula de hierro" que Max Weber temía ya hace cerca de cien años.

De una u otra manera, probablemente tengamos que aprender a pensar más en términos de personas y menos en términos de reglas e instituciones. Un enfoque multiagencia de la seguridad de la comunidad resulta absolutamente necesario si tenemos que enfrentarnos con algunas de las causas más estructurales de la inseguridad, pero no deben multiplicarse los formalismos ni los problemas mutuos de comunicación que sufren todas las instituciones actualmente implicadas. Quién lleva a cabo qué es de

${ }^{19}$ Bruggen Bouwen - Onderzoek Integratiebeleid, Tweede Kamer, 2003-2004, 28 689, nrs.8-9 (637 pp.)

Revista Española de Investigación Criminólogica

REIC AC-02-05 http://www.criminologia.net

ISSN 1696-9219 
importancia secundaria, y la forma en que con frecuencia la policía, los trabajadores sociales y las administraciones locales se pasan la pelota tiene que terminar. El criminólogo belga Peter Goris (2000) ha mostrado que la falta de cooperación entre diferentes actores es el principal problema de la política de seguridad ciudadana. No resulta nada fácil dar con la receta sobre cómo todos estos socios podrían cooperar en un modo más constructivo, pero la investigación de Goris ofrece un punto de partida. En primer lugar muestra que contar con las personas adecuadas en el sitio adecuado es más importante que cualquier brillante sustrato filosófico. En segundo lugar, Goris concluye que un modelo conflictual entre socios, dónde cada uno enfoca la cuestión de la seguridad de la comunidad desde el ángulo de su disciplina es más fructífero que un consenso forzado marcado por las prioridades policiales.

\section{Conclusión}

Durante la última década, las ideas y proyectos sobre seguridad ciudadana han cambiado de manera fundamental. En los proyectos iniciales, la prevención del delito se suponía que debía tener lugar fuera del ámbito de la justicia penal y estar dirigida a afrontar las causas (principalmente sociales) del delito. Hoy por hoy, la racionalidad penal ha permeado prácticamente todas las medidas de prevención del delito. "Prevención" significa ahora, principalmente, intervención proactiva sobre la base de perfiles de riesgo. El destierro es la nueva metáfora de esta política de seguridad pública y los miedos de los ciudadanos que observan la ley son la fuerza motriz que se encuentra detrás. En este artículo he intentado analizar por qué se ha producido dicho cambio.

Lo que debemos temer es que el control del delito se esté tornando en una lucha sin rostro social, en la que los grupos más desaventajados y con menos poder de la sociedad se conviertan en víctimas. Hubo un tiempo en que se suponía que la política de seguridad ciudadana era modo de luchar contra sus privaciones, pero ahora más bien parece contribuir a generar una sociedad cada vez más polarizada.

Por supuesto las cuestiones que surgen en torno a este tema son lo suficientemente complejas como para no presentar aquí conclusiones finales. Por ello mis conclusiones deben leerse básicamente como puntos para ser tenidos en cuenta en el debate futuro, un debate que espero haya dado un pequeño paso adelante.

\section{BIBLIOGRAFIA}

Beijerse, Jolande uit \& René van Swaaningen (1993) 'El Control Social como Política Estatal: Moralismo Pragmático con Déficit Estructural. La falacia de la prevención del delito en los Países Bajos', en: Roberto Bergalli (ed.), Sistema Penal e Intervenciones Sociales; algunas experiencias nacionales, regionales y locales en Europa. Barcelona: Hacer, pp.177-219

Beijerse, Jolande uit \& René van Swaaningen (2003) 'Recluso en Holanda', en: Panoptico, no.5, pp.78-92

Body-Gendrot, Sophie (2004) 'Stedelijk geweld in Frankrijk', en: Justitiële Verkenningen vol.30, no.7, pp.69-80.

Boutellier, Hans (2004) The Safety Utopia; contemporary discontent and desire as to crime and punishment. Dordrecht: Kluwer. 
Brants, Kees (1986) 'Criminaliteit, politiek en criminele politiek; de Haagse receptie van een nieuwe heilsleer', in: Tijdschrift voor Criminologie, vol.28, pp.219-235.

Brink, Gabriël van den (2002) Mondiger of moeilijker? Een studie naar de politieke habitus van hedendaagse burgers. Den Haag: Sdu.

Bunt, Henk van de \& René van Swaaningen (2005) 'Privatisering van de veiligheidszorg', in: Laurens W. Winkel, Jacques J.M. Jansen a.o. (eds.) 'Privatisering van de veiligheidszorg'. The Hague: BJU.

Christie, Nils (1993) La industria del control del delito. ¿La nueva forma del holocausto?, Buenos Aires: Ed. Del Puerto [Crime Control as Industry; towards gulags Western style? London: Routledge]

Corstens, Geert J.M. (2005) 'Dijkdoorbraken in de strafrechtspleging', in: Nederlands Juristen Blad no.6, 11 February 2005.

Crawford, Adam (1997) The Local Governance of Crime; appeals to community and partnerships. Oxford: Clarendon.

Downes, David (1988) Contrasts in Tolerance; post-war penal policy in The Netherlands and England and Wales. Oxford: Clarendon.

Elchardus, Mark (2002) De dramademocratie. Tielt: Lannoo.

Garland, David (2001) The Culture of Control; crime and social order in contemporary society. Oxford University Press.

Gemeente Rotterdam (2002a) Vijfjarenactieprogramma Veiligheid Rotterdam. Rotterdam: Programmabureau Veilig.

Gemeente Rotterdam (2002b) Het nieuwe elan van Rotterdam... en zo gaan we dat doen. Rotterdam: College van B\&W.

Goris, Peter (2000) Op zoek naar de krijtlijnen van een sociaal rechtvaardige veiligheidszorg; analyse van relaties tussen professionele actoren in het kader van een geïntegreerde preventieve aanpak van veiligheidsproblemen in achtergestelde woonbuurten, thesis KU Leuven.

Haan, Willem de (1990) The Politics of Redress; crime, punishment and penal abolition.. London: Unwin Hyman.

Haan, Willem de (1995) 'Integrale veiligheid; beleidsvernieuwing of beleidsvervaging?', Justitiële Verkenningen 21 ste jrg., nr.5, pp.25-48.

Hebberecht, Patrick \& Dominique Duprez (eds.) The Prevention and Security Policies in Europe. Brussels: VUB University Press. 
Hörnqvist, Magnus (2004) 'Veiligheid en overheidsgeweld', en: Justitiële Verkenningen vol.30, no.7, pp.41-50.

Hughes, Gordon \& Adam Edwards (eds.) (2002) Crime Control and the Community; the new politics of public safety. Cullompton: Willan.

Jones, Trevor \& Tim Newburn (2004) 'The Convergence of US and UK Crime Control Policy; exploring substance and process', in: Newburn \& Sparks, 2004, pp. 123-151.

Kelling, George L. \& Catherine M. Coles (1996) ¡No más ventanas rotas!, México: Instituto Cultural Ludwig von Mises. [Fixing broken Windows; restoring order \& reducing crime in our communities. New York: The Free Press].

Mak, Geert (2005) Gedoemd tot kwetsbaarheid. Amsterdam: Atlas.

MvJ (2002) Ministerie van Justitie, Naar een veiliger samenleving. On line (en holandés): www.justitie.nl.

Newburn, Tim \& Richard Sparks (eds.) Criminal Justice and Political Cultures; nationaland international dimensions of crime control. Cullompton: Willan.

Presdee, Mike (2000) Cultural Criminology and the Carnival of Crime. London: Routledge.

Stangeland, Per (2004) 'Openbare orde in Spanje sinds 11 maart 2004', en: Justitiële Verkenningen vol.30, no.7, pp.51-68.

Storm, Servaas \& Ro Naastepad (2003) 'The Dutch Distress', in: New Left Review, vol.20, March-April, pp.131-151

Swaaningen, René van \& Jolande uit Beijerse (1993) 'Las Paradojas en el Sistema Penal. El Debate sobre la Despenalización y las Medidas Alternativas en los Países Bajos', en: Delito y Socieded; revista de ciencias sociales, vol.2, no.3, pp.55-84

Swaaningen, René van (2002) 'Hacia un nuevo concepto de seguridad comunitaria, in: Revista Catalana de Seguritat Pública (número especial sobre políticas de seguridad y prevención en Europa durante los años noventa), no.11, pp. 221-243

Swaaningen, René van (2003) 'La demanda de seguridad ciudadana; la política populista y el debate sobre "vivilidad", en: Archipiélago; cuadernos de crítica de la cultura, no.55, pp.75-81

Swaaningen, René van (2004) 'Sweeping the street clean; Rotterdam's ideas on 'community safety' in 2004', paper GERN Justice and Community Seminar, 3-4 November 2004, UC Dublin

Uitermark, Justus \& Jan Willem Duyvendak (2004) 'Civilising the European city; revanchist urbanism in Rotterdam, the Netherlands' (unpublished manuscript Amsterdam School for Social Science Research) 
WRR (2003) Waarden, normen en de last van het gedrag; rapport van de Wetenschappelijke Raad voor het Regeringsbeleid. Amsterdam: Amsterdam University Press.

Young, Jock (1999) La sociedad "excluyente": exclusión social, delito y diferencia en la modernidad tardía. Madrid: Marcial Pons. [The Exclusive Society; social exclusion, crime and difference in late modernity. London: Sage.]

\section{EL AUTOR}

René van Swaaningen es Senior Lecturer en Criminología de la Erasmus University Rotterdam (EUR) y director del Programa de BA y MA en criminología. Es también profesor invitado de varias universidades europeas y latino-americanas. Su formación en criminología (Master) comenzó con una tesis teórica relativa a las relaciones de poder y el control social, y su tesis doctoral (1995) versó sobre la reelaboración teórica de la criminología crítica europea. Es (co-)autor de ocho libros y ha publicado más de cien artículos, principalmente sobre política criminal, criminología comparada, penología y prevención del delito y teoría de la justicia penal y la criminología. En la actualidad trabaja en un libro sobre seguridad comunitaria en la sociedad de riesgo 